\title{
High-yield parallel transfer print integration of III-V substrate- illuminated C-band photodiodes on silicon photonic integrated circuits
}

\author{
Grigorij Muliuk*,a, b , Jing Zhanga, b, Jeroen Goyvaerts $^{\mathrm{a}, \mathrm{b}}$, Sulakshna Kumari ${ }^{\mathrm{a}, \mathrm{b}}$, Brian Corbett ${ }^{\mathrm{c}}$, \\ Dries Van Thourhout ${ }^{\mathrm{a}, \mathrm{b}}$, Günther Roelkens ${ }^{\mathrm{a}, \mathrm{b}}$ \\ aPhotonics Research Group, Ghent University-imec, Technologiepark-Zwijnaarde 15, 9052 Ghent, Belgium; \\ ${ }^{\mathrm{b}}$ Center for Nano- and Biophotonics (NB-Photonics), Ghent University, Belgium; \\ ${ }^{\mathrm{c}}$ Tyndall National Institute, Cork T12R5CD, Ireland.
}

\begin{abstract}
Transfer printing is an enabling technology for the efficient integration of III-V semiconductor devices on a silicon waveguide circuit. In this paper we discuss the transfer printing of substrate-illuminated III-V C-band photodetectors on a silicon photonic waveguide circuit. The devices were fabricated on an InP substrate, encapsulated and underetched in $\mathrm{FeCl}_{3}$, held in place by photoresist tethers. Using a $2 \times 2$ arrayed PDMS stamp with a pitch of $500 \mu \mathrm{m}$ in $\mathrm{x}$-direction and $250 \mu \mathrm{m}$ in y-direction the photodiodes were transfer printed onto DVS-BCB-coated SOI waveguide circuits interfaced with grating couplers. 83 out of 84 devices were successfully integrated.
\end{abstract}

Keywords: Heterogeneous integration, photodiodes, photonic integration, transfer printing, silicon photonics.

\section{INTRODUCTION}

Heterogeneous III-V-on-Si integration for photonics has been an intense research topic in recent years. With the intention to integrate an efficient laser source on silicon photonic integrated circuits (PICs), several integration approaches such as direct III-V growth [1] and die-to-wafer bonding [2-4] are being studied, however a compelling industrial wafer scale technique is still unavailable.

Transfer printing is a novel pick-and-place technology whereby devices processed in a dense array on the III-V wafer can be selectively picked using a polydimethylsiloxane (PDMS) stamp and printed on a silicon photonics wafer with high alignment accuracy (better than $\pm 1 \mu \mathrm{m}, 3 \sigma[5]$ ). Recently, several demonstrations of this technology have been reported, including transfer printing III-V photodiodes [6] and lasers [7-9] but also PbS quantum dot films [10], Si-Ge devices [11], etc., showcasing the versatility of the technology.

The main advantage of the transfer printing is the parallel integration of the III-V components without requiring large bond pads as are needed for flip-chip integration. This enables a time- and material-efficient integration of III-V devices on $200 \mathrm{~mm}$ or $300 \mathrm{~mm}$ silicon photonics wafers in several printing cycles. This paper is dealing with transfer printing arrays of III-V substrate illuminated C-band photodiodes on silicon photonics waveguide circuits. Using a $2 \times 2$ array stamp we picked and printed 84 devices. We present the successful fabrication, release, transfer printing, post-processing and characterization, obtaining a yield of $98.8 \%$.

\section{DESIGN AND FABRICATION}

\subsection{Design}

The silicon photonic target wafers were fabricated in imec's passive technology platform using a $220 \mathrm{~nm}$ thick silicon device layer, a $1.2 \mu \mathrm{m}$ thick top oxide cladding and $70 \mathrm{~nm}, 150 \mathrm{~nm}$ and $220 \mathrm{~nm}$ etch depths [12]. The schematic layout of the target waveguide circuit is displayed in Fig. 1 a). The circuit consists of arrays of 6x14 C-band input and output grating couplers, placed with a $500 \mu \mathrm{m} / 250 \mu \mathrm{m}$ pitch in $\mathrm{x}$ - and y-direction. The top 42 grating couplers are $1 \mathrm{D}$ grating couplers $(4.5 \mathrm{~dB}$ single mode fiber-to-chip loss per coupler at $1550 \mathrm{~nm})$ and the bottom 42 are $2 \mathrm{D}$ grating couplers $(6 \mathrm{~dB}$ single mode fiber-to-chip loss per coupler at $1550 \mathrm{~nm}$ ). Tetris-brick alignment markers were added to aid to the high printing alignment accuracy as will be discussed below. 
On the III-V source wafer, $55 \times 55 \mu \mathrm{m}$ square photodiodes with $23 \mu \mathrm{m}$ circular apertures were designed in a dense array on a pitch of $100 \mu \mathrm{m}$ and $125 \mu \mathrm{m}$ in $\mathrm{x}$ - and y-direction respectively (Fig $1 \mathrm{~b}$ )). The photodiodes are to be released and transfer printed using a $2 \times 2$ arrayed stamp spaced with a $500 \mu \mathrm{m} \times 250 \mu \mathrm{m}$ pitch.

a)

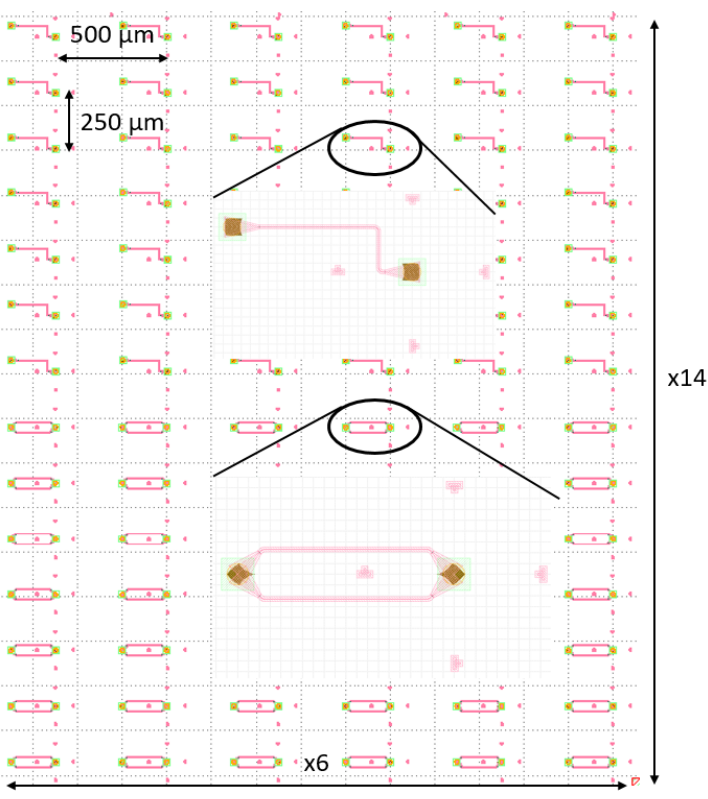

b)

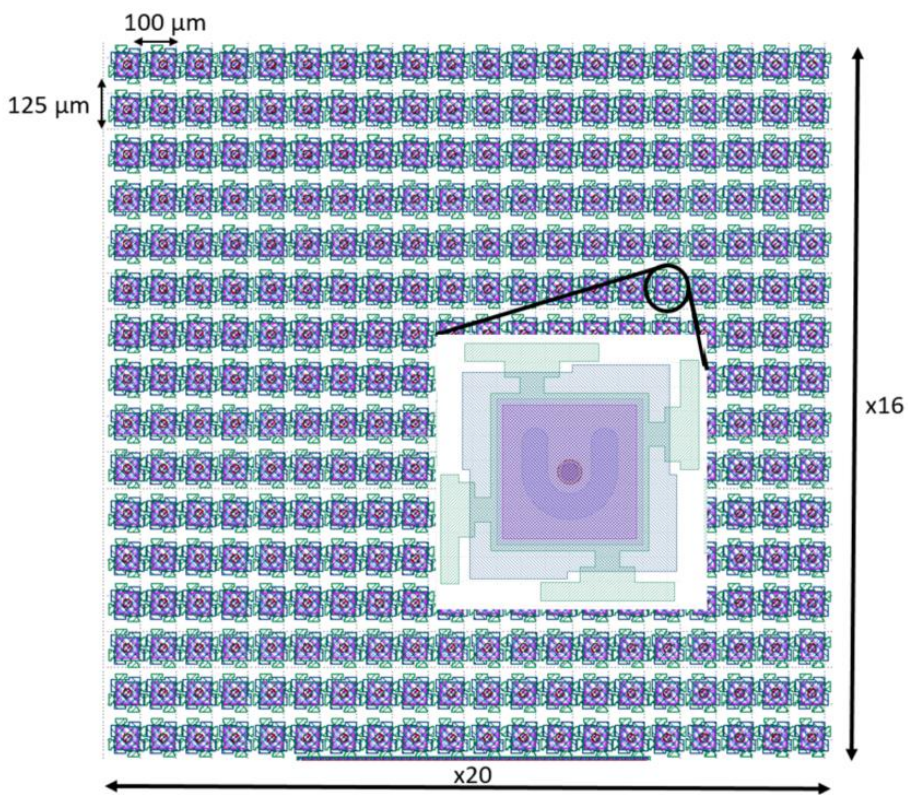

Fig. 1. a) Schematic layout of the silicon photonic target wafer; b) schematic layout of the III-V source wafer.

\subsection{Photodiode Fabrication}

The epitaxial material stack of the III-V photodiodes is presented in Table 1. The p-i-n layer stack consists of an intrinsic InGaAs absorber sandwiched between InP p-type and n-type cladding layers and an additional bottom InGaAs layer which acts as a release layer. The p-contact is formed on a heavily doped InGaAs layer.

Table 1. Epitaxial III-V layer stack for photodiode fabrication.

\begin{tabular}{|l|l|l|c|l|}
\hline Layer & Material & $\begin{array}{l}\text { Thickness } \\
(\mathrm{um})\end{array}$ & Doping & Function \\
\hline 1 & InP & 100 & n.i.d & Cap \\
\hline 2 & InGaAs & 100 & $>1 \times 10^{19}$ & p-contact \\
\hline 3 & InGaAs & 200 & $1 \times 10^{19}$ & p-contact \\
\hline 4 & InP & 300 & $5 \times 10^{17}$ & p-contact \\
\hline 5 & InGaAs & 1000 & n.i.d & Absorption \\
\hline 6 & InP & 240 & $1 \times 10^{18}$ & n-contact \\
\hline 7 & InP & 60 & n.i.d. & n-contact \\
\hline 8 & InGaAs & 1000 & n.i.d. & Release \\
\hline 9 & InP & 150 & n.i.d. & Buffer/Substrate \\
\hline 10 & InP & & n-type & Substrate \\
\hline
\end{tabular}


a)

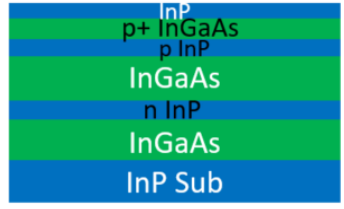

e)

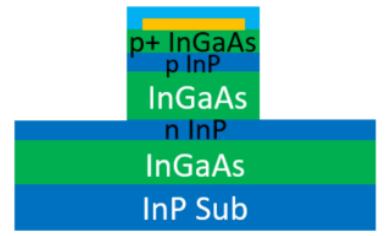

i)

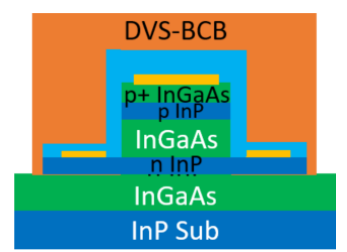

b)

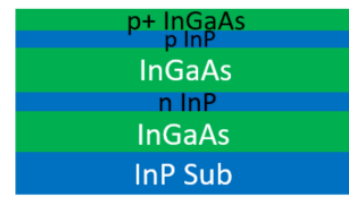

f)

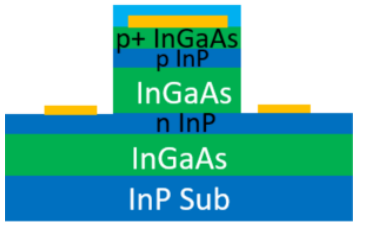

j)

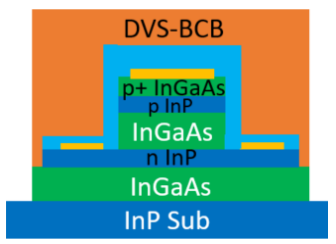

c)

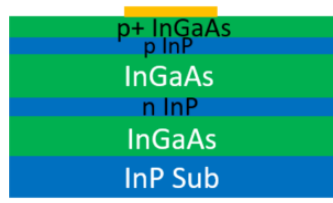

g)

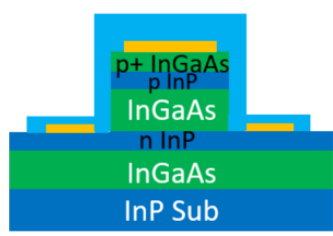

k)

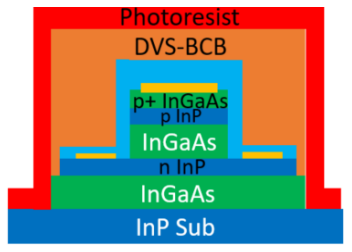

d)

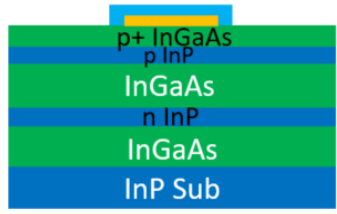

h)

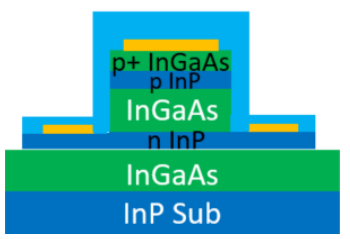

1)

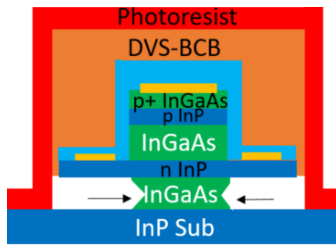

Fig. 2. Schematic illustration of III-V photodiode fabrication, release processes.

III-V photodiode fabrication was performed in the Ghent University cleanroom and the full sequence is schematically depicted in Fig. 2. We start with the full layer stack (Fig. 2 a)) and used $\mathrm{HCl}$ wet etching for about 20 seconds to remove the InP cap layer (Fig. 2 b)). Then, circular $21 \mu \mathrm{m}$ diameter Ti/Au p-contacts were deposited (Fig. 2 c)). By depositing and patterning a $150 \mathrm{~nm}$ thick plasma-enhanced chemical vapor deposition (PECVD) mixed frequency silicon nitride (MF$\mathrm{SiNx}$ ), the p-metal was protected (Fig. $2 \mathrm{~d}$ )), using the layer as a hard to mask to define the $23 \mu \mathrm{m}$ circular mesa by etching it using inductively coupled plasma (ICP) using $\mathrm{CH}_{4} / \mathrm{H}_{2}$ for $40 \mathrm{~min}$. The etch was stopped on top of the $\mathrm{n}$-InP layer and the residual intrinsic InGaAs was removed by dipping the sample into a Piranha 1:1:10 solution for 30 seconds (Fig. 2 e)). Then, the U-shape Ni/Ge/Au n-contacts (Fig. $2 \mathrm{f}$ )) and an additional $150 \mathrm{~nm}$ of MF-SiNx, which was patterned in square patterns of 50 x $50 \mu \mathrm{m}$ was defined (Fig. $2 \mathrm{~g}$ )). This SiNx pattern was used as a hard mask to etch the n-InP layer and stop on the InGaAs release layer using a combination of ICP dry etching and $\mathrm{HCl}: \mathrm{H}_{2} \mathrm{O}$ 1:1 wet etching for 45 seconds (Fig. 2 h)). $\sim 1.5 \mu \mathrm{m}$ divinyl-siloxane-bis-benzocyclobutene (DVS-BCB) passivation was spin-coated, cured at $280^{\circ} \mathrm{C}$ and patterned (Fig. $2 \mathrm{i}$ )). Afterwards the release layer was patterned using ICP (Fig. $2 \mathrm{j}$ )) and the device was encapsulated using a $\sim 3 \mu \mathrm{m}$ thick photoresist. After patterning the photoresist tethers (Fig. $2 \mathrm{k}$ )) the release was performed by placing the devices in an $\mathrm{FeCl}_{3}: \mathrm{H}_{2} \mathrm{O}(1 \mathrm{mg}: 2 \mathrm{ml})$ solution at $3^{\circ} \mathrm{C}$ for about 55 minutes to underetch the InGaAs release layer and make the devices free hanging, anchored to the substrate by the photoresist tethers (Fig. 2 1)). The top view of a fabricated and released $20 \times 7$ array of photodiodes is depicted in Fig. 3.

\subsection{Transfer Printing}

Transfer printing was performed using the $\mu \mathrm{TP}-100$ lab scale printer using the automatic printing mode. We used square $60 \times 60 \mu \mathrm{m}$ PDMS posts in a $2 \times 2$ array on a pitch of 500 by $250 \mu \mathrm{m}$. The process is schematically depicted in Fig. 4 . Devices are picked when the stamp is laminated with the released devices and quickly moves up (Fig. 4 a)). In this case the tethers break at their weakest points and devices are attached to the stamp (Fig 4 b)). For printing, the stamp is brought in close proximity of the target wafer and the devices are first aligned with respect to the target wafer (Fig. 4 c)). Printing is performed by laminating the device against the $\sim 50 \mathrm{~nm}$ thick DVS-BCB spin-coated target wafer and slowly moving the stamp in the vertical direction (Fig. 4 d)). This way, the devices stay attached to the target substrate. 


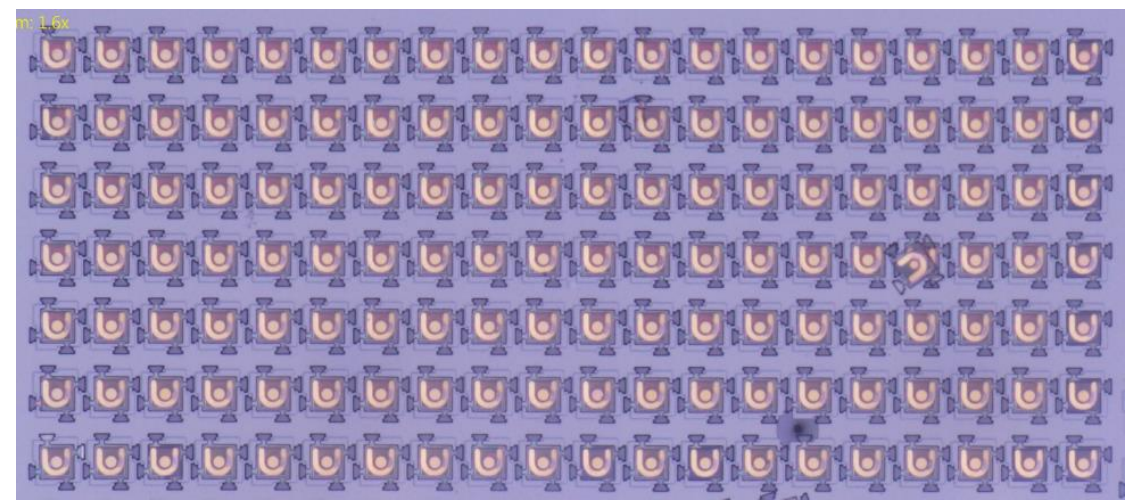

Fig. 3. Top view of the fabricated and released $20 \times 7$ array of C-band photodiodes.

a)

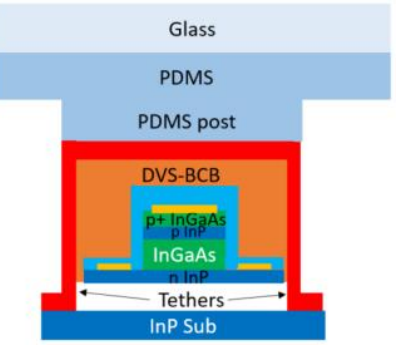

b)

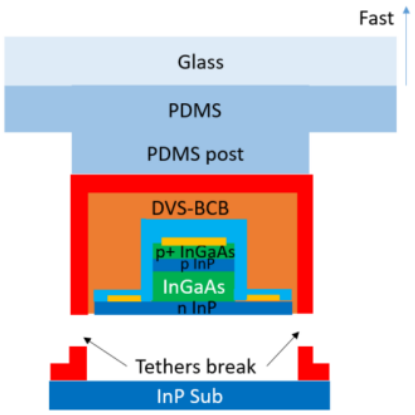

c)

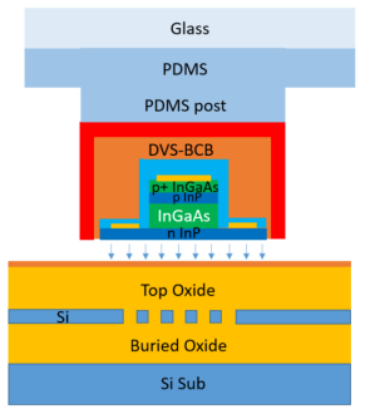

d)

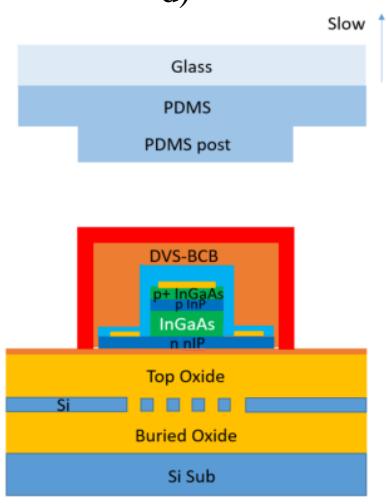

Fig. 4. Schematic sequence of the transfer printing process.

Fig. 5 shows the source substrate after the transfer printing. Arrayed transfer printing was performed in an automated mode, skipping the positions of collapsed or broken devices (marked in red in Fig. 5). 84 devices were picked using the $2 \times 2$ arrayed printing and 80/84 devices were printed. The 4 failed devices were populated on the target wafer by picking and printing using a 1x1 PDMS stamp (shown in blue in Fig. 5), as a rework procedure.

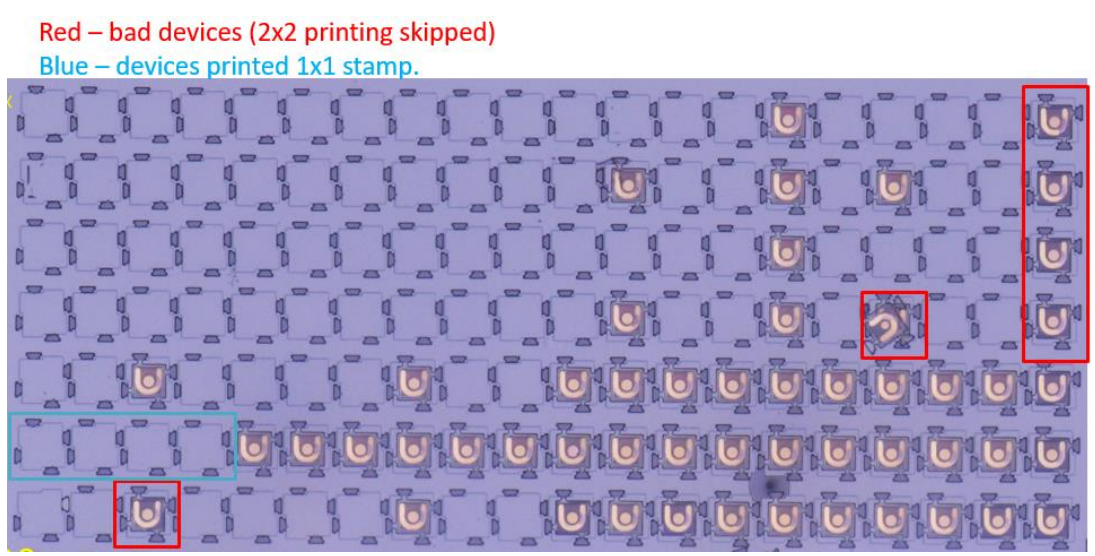

Fig. 5. Top view of the source wafer after completing the transfer printing. Collapsed or broken devices are shown in red which were skipped by the $2 \times 2$ array stamp. The blue rectangle indicates devices that were picked and printed in 1x1 mode. 


\subsection{Post-Processing}

After transfer printing arrays of devices on the target wafer, the photoresist encapsulation was reflown at $140^{\circ} \mathrm{C}$ for 10 minutes to improve III-V device adhesion and was then removed using $\mathrm{O}_{2}$ reactive ion etching (RIE) (Fig. 6 a)). The DVSBCB was fully cured at $280^{\circ} \mathrm{C}$ after which a new thick $\sim 3 \mu \mathrm{m}$ DVS-BCB layer was spin coated and cured at $280^{\circ} \mathrm{C}$ again (Fig. 6 b)). Then using RIE ( $\mathrm{SF}_{6} / \mathrm{O}_{2}$ gas mixture for $\sim 6$ minutes), the DVS-BCB layer was etched back and the p- and nelectrode were opened (Fig. $6 \mathrm{c}$ )). After this $\sim 1 \mu \mathrm{m}$ thick Au ground-signal-ground tracks were defined for electrical device characterization (Fig. 6 d)).

a)

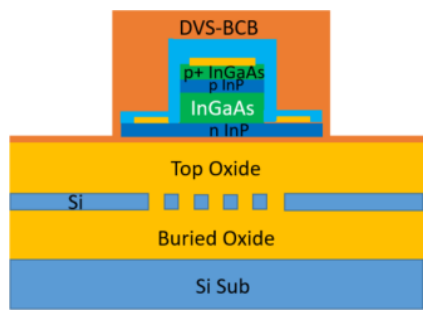

b)

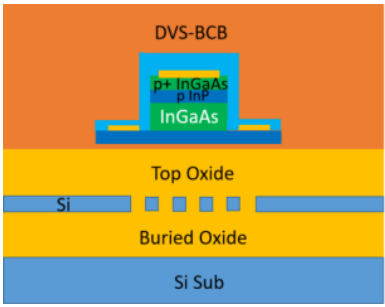

c)

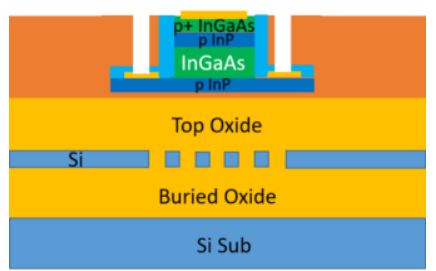

d)

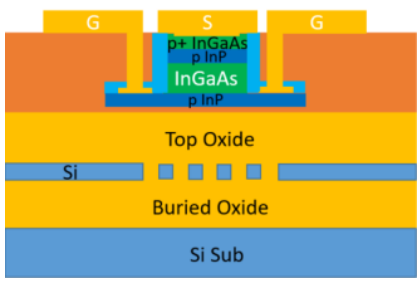

Fig. 6. Schematic illustration of the post-processing of the target wafer.

The target chip, after completing the post-processing is depicted in Fig. 7. All devices were printed and metalized. Only one device on row 13, column 2 transfer printed with the noticeable misalignment on the target (see the left inset in Fig. 7).

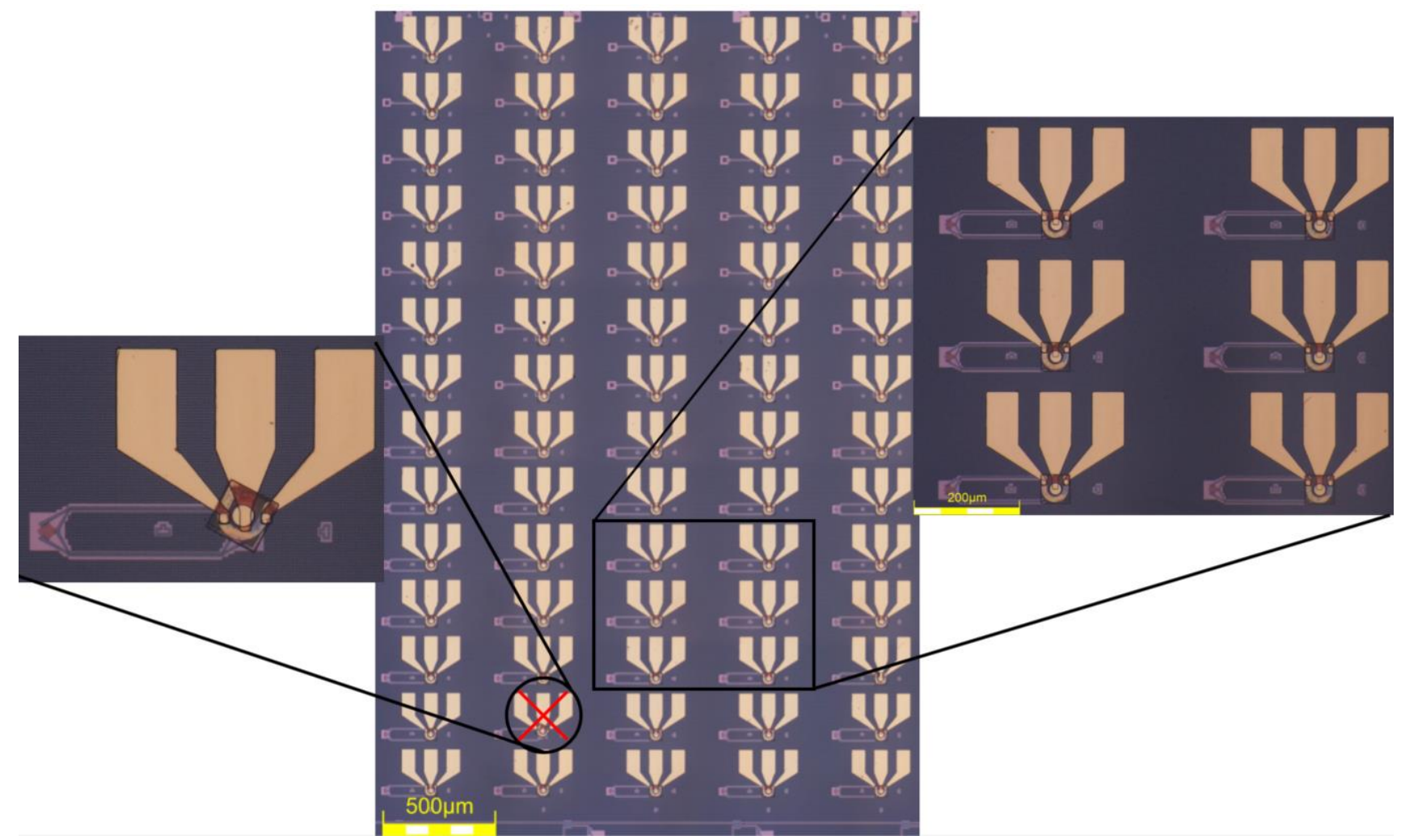

Fig. 7. Transfer printed bottom-illuminated devices after completing the post-processing. The inset on the left depicts the only device transfer printed with poor alignment accuracy. 


\section{DEVICE CHARACTERIZATION}

\subsection{IV characteristics}

The IV characteristics of all 84 integrated devices were measured using DC probes. A typical IV of one device is shown in Fig. 8 a). All 84 devices show comparable IV characteristics with an average series resistance of $79 \Omega$ (standard deviation $3 \Omega$ ) and an average dark current of $0.9 \mu \mathrm{A}$ at $-1 \mathrm{~V}$ bias (standard deviation $0.3 \mu \mathrm{A}$ ). The histogram of series resistance and dark current are plotted in Fig. 8 b) and c) respectively.

a)

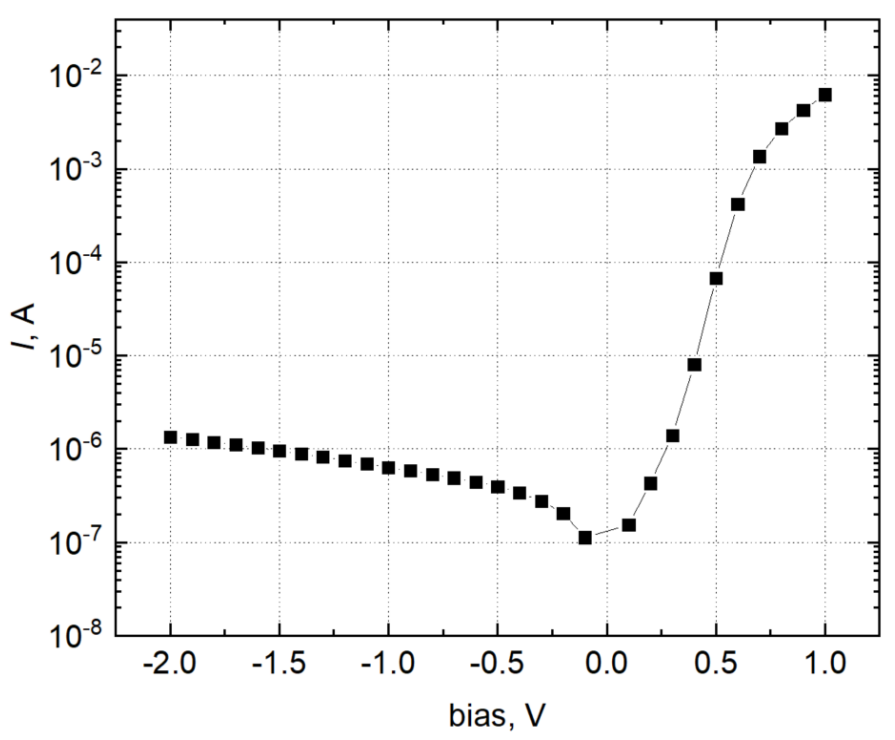

b)

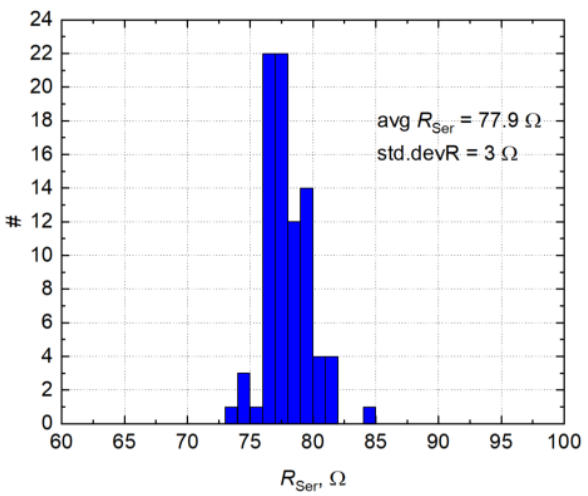

c)

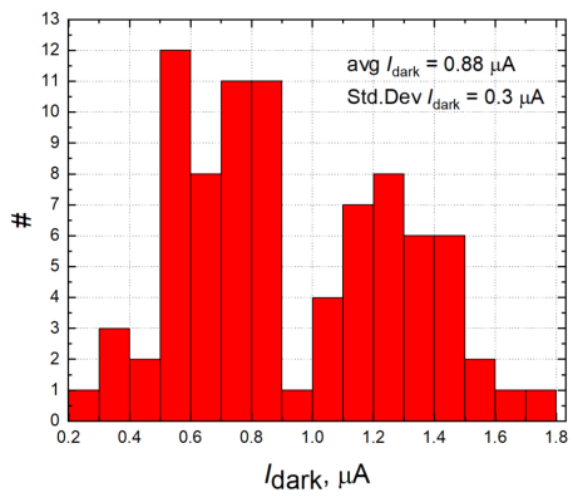

Fig. 8. a) A typical IV curve of one transfer printed photodiode; b) histogram of the series resistance of all 84 transfer printed photodiodes; c) histogram of the dark current of all 84 transfer printed photodiodes.

\subsection{Responsivity}

Using a Santec TSL510 tunable C-band laser and a 10 degree tilted single mode fiber, light was coupled into the silicon photonics chip (using a 1D or 2D grating coupler). The waveguide-referred responsivity of the 42 devices integrated on 1D grating couplers and its dependence on wavelength for a typical device is presented in Fig. 9 a). The responsivity increases with wavelength. This increase can be attributed to the increase of the directionality of the 1D grating coupler at longer wavelengths. Fig. 9 b) and c) display the histograms of waveguide-referred responsivities of all 42 devices integrated on a $1 \mathrm{D}$ grating coupler at $1550 \mathrm{~nm}$ and $1570 \mathrm{~nm}$ respectively. 
b)

a)

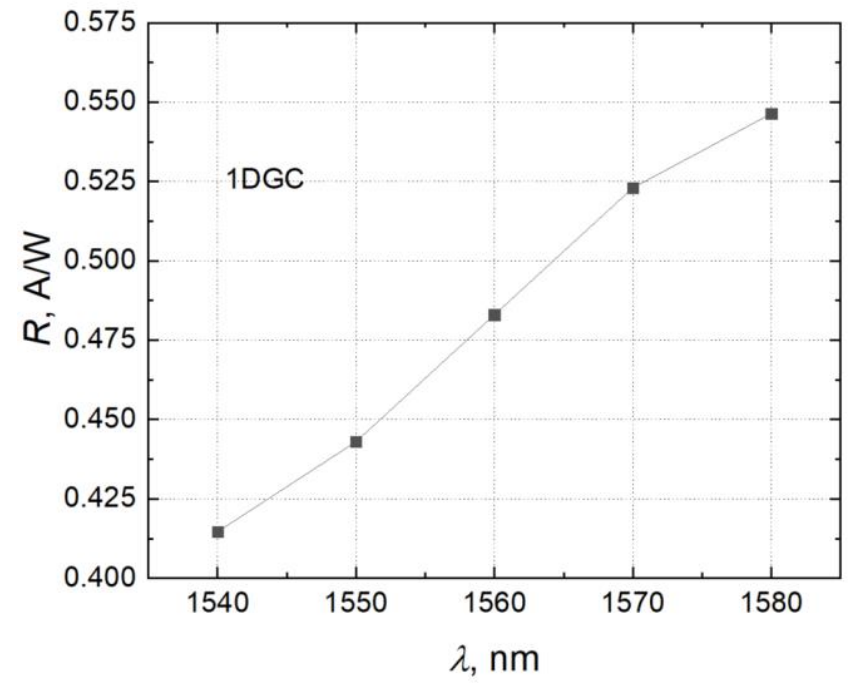

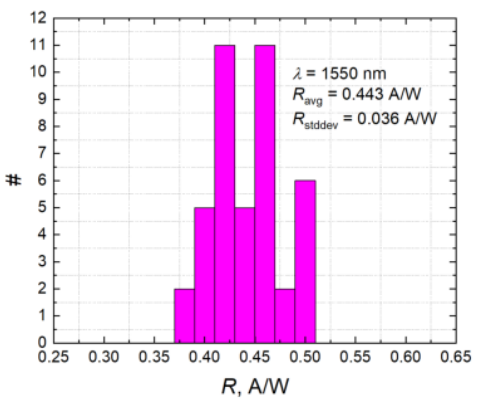

c)

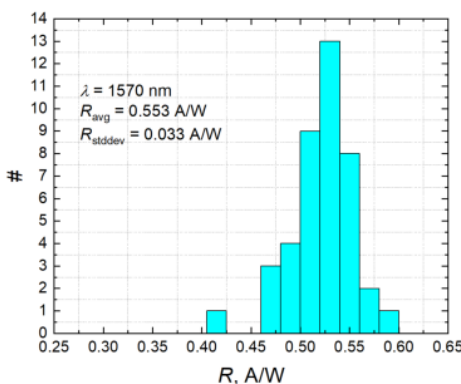

Fig. 9. a) Waveguide-referred responsivity for a typical device transfer printed on a 1D grating coupler. Histograms of the waveguide-referred responsivity at $1550 \mathrm{~nm} \mathrm{~b}$ ) and at $1570 \mathrm{~nm} \mathrm{c}$ ) for the 42 photodiodes transfer printed on a 1D grating coupler.

a)

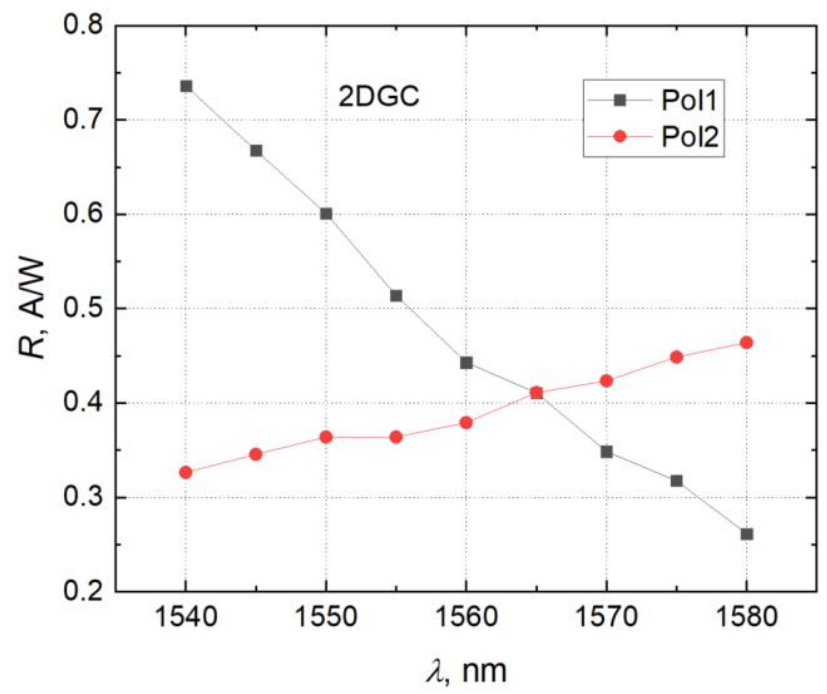

b)

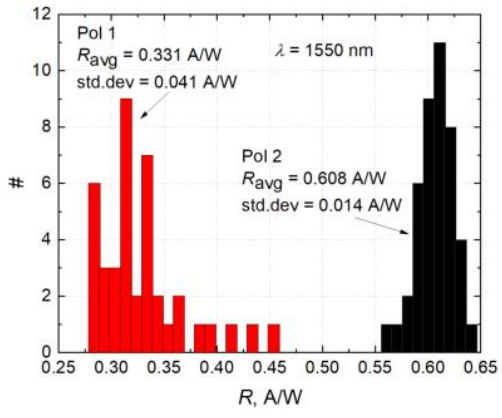

c)

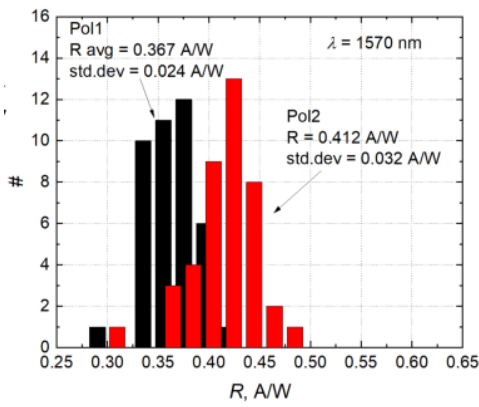

Fig. 10. a) Waveguide-referred responsivity for a typical device integrated on $2 \mathrm{D}$ grating coupler measured for both states of polarizations. b) histogram of responsivities at $1550 \mathrm{~nm}$ at polarization state 1 and 2; c) histogram of responsivities at $1570 \mathrm{~nm}$ at polarization state 2 . 
The waveguide-referred responsivity for photodiodes integrated on 2D grating couplers measured for two polarization states corresponding to the maximum and minimum response (denoted as Pol1 and Pol2) and its wavelength dependence is shown in Fig. 10.a). This behavior is not well understood at the moment and numerical simulations are necessary to understand these dependencies better. Fig. $10 \mathrm{~b}$ ) and c) show the histogram for the waveguide-referred responsivity at $1550 \mathrm{~nm}$ and $1570 \mathrm{~nm}$ for polarization states 1 and 2 respectively for all 41 devices (the device printed with the noticeable misalignment was not taken into account).

\subsection{Small Signal Measurement}

The small signal response was measured using a vector network analyzer (VNA) using a Santec TSL510 tunable C-band laser and Mach-Zehnder modulator. The $\mathrm{S}_{21}$ parameter for different photodiode bias is displayed in Fig. 11 a). The histogram of the $3 \mathrm{~dB}$ bandwidth of all the 83 transfer printed devices ( $3 \mathrm{~V}$ reverse bias) is shown in Fig. $11 \mathrm{~b}$ ). The $3 \mathrm{~dB}$ bandwidth spans between 12 and $14.4 \mathrm{GHz}$ with an average value of $13.5 \mathrm{GHz}$.

a)

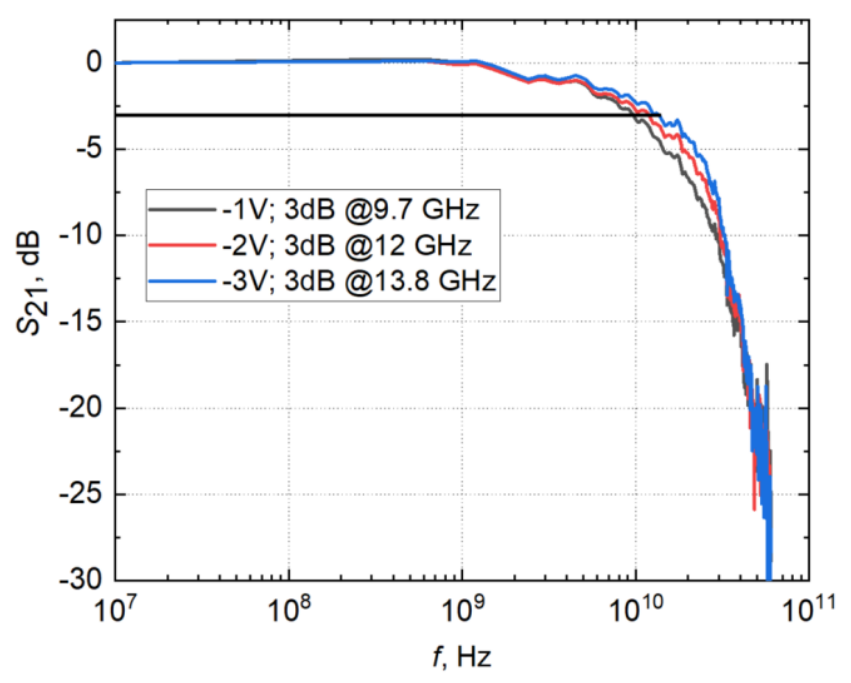

b)

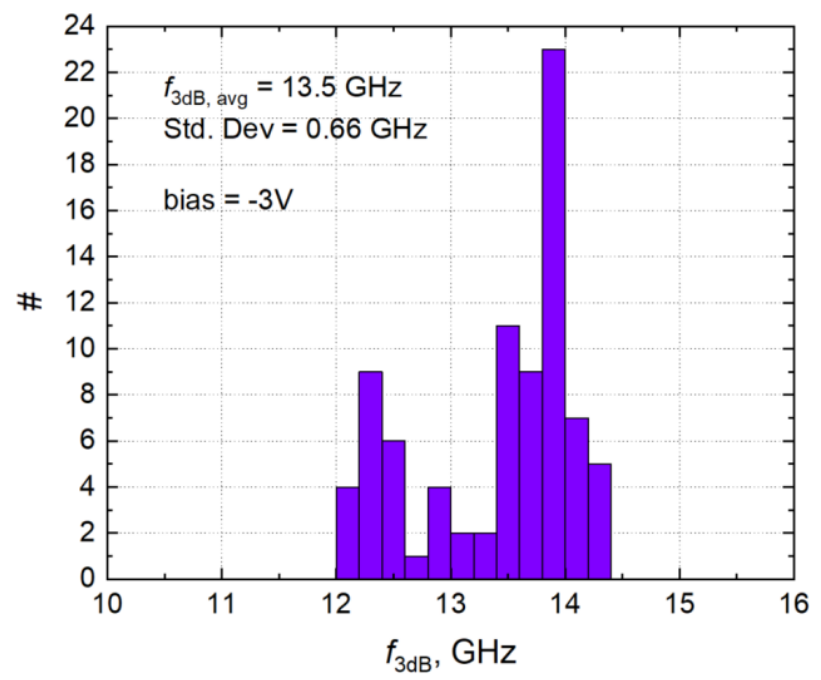

Fig. 11. a) S21 curve measured for one representative printed photodiode at different bias voltages. b) Histogram of $3 \mathrm{~dB}$ bandwidths of 83 photodiodes, measured at $-3 \mathrm{~V}$ bias.

\section{CONCLUSIONS}

In this paper we demonstrated parallel transfer printing of substrate-illuminated III-V C-band photodetectors. We developed the photodiode fabrication and release processes, as well as the automated picking and printing of the devices. This demonstration showcases the advantages of transfer printing technology for scalable III-V-on-Si integration.

\section{REFERENCES}

[1] Shi, Y. Wang, Z. Van Campenhout, J. Pantouvaki, M. Gou, W. Kunnert, B. van Thourhout, D. "Optical pumped InGaAs/GaAs nano-ridge laser epitaxially grown on a standard 300-mm Si wafer", Optica, 4(12), 1468-1473 (2017).

[2] Roelkens, G. Abbasi, A. Cardile, P. Dave, U. de Grote, A. de Koninck, Y. Dhoore, S. Fu, X. Kumari, S. Keyvaninia, S. Kuyken, B. Li, L. Mechet, P. Munneb, M. Sanchez, D. Shao, H. Spuesens, T. Subramanian, A. Z. Uvin, S. Tassaert, M. van Gasse, K. Verbist, J. Wang, R. Wang, Z. Zhang, J. van Campenhout, J. Bauwelinck, J. Morthier, G. Baets, R. van Thourhout, D. "III-V-on-silicon photonic devices for optical communication and sensing", Photonics (invited), 2(3), 969-1004 (2015).

[3] Ramdane, A. Ougazzaden, A. Devaux, F. Delorme, F. Schneider, M. and Landreau, J. "Very simple approach for high performance DFB laser electroabsorption modulator monolithic integration," Electronics Letters, 30(23), 1980-1981 (1994). 
[4] Abassi, A. Verbist, J. Shimamin, L. A. Verplaetse, M. De Keulenaer, T. Vaernawyck, R. Pierco, R. Vyncke, A. Yin, X. Torfs, G. Morthier, G. Bauwelink, J. Roelkens, G. "IEEE Photonics Technology Letters", 30 (15), 1095-1098 (2018).

[5] Gomez, D. Ghosal, K. Meitl, M. A. Bonafede, S. Prevatte, C. Moore, T. Raymond, B. Kneeburg, D. Fecioru, A. Trindade, A. J. Bower, C. A. "Process Capability and Elastomer Stamp Lifetime in Micro Transfer Printing". IEEE 66th Electronic Components and Technology Conference, Las Vegas, NV, 681-687 (2016).

[6] Zhang, J. De Groote, A. Abbasi, A. Loi, R. O'Callaghan, J. Corbett, B. Trindade, A. J. Bower, C. A. Roelkens, G. “A Silicon Photonics Fiber-To-The-Home Transceiver Array based on Transfer-Printing-Based Integration of III-V Photodetectors", Optics Express, 25(13), 14290-14299 (2017).

[7] Justice, J. Bower, C. Meitl, M. Mooney, M. B. Gubbins, M. A. Corbett, B. "Wafer-scale integration of group III-V lasers on silicon using transfer printing of epitaxial layers", Nature Photonics, 6, 610-614 (2012).

[8] Loi, R. Roycroft, B. O'Callaghan, J. Justice, J. Gocalinska, A. Peluchi, E. Trindade, A. J. Bower, C. A. Roelkens, G. Corbett, B. "Micro-transfer printing for advanced scalable hybrid photonic integration", European Conference on Integrated Optics, Valencia, Spain, 98-100 (2018).

[9] Juvert, J. Cassese, T. De Groote, A. Snyder, B. Bogaerts, L. Jamieson, G. Van Campenhout, J. Roelkens, G. Van Thourhout, D. "Integration of etch facet, electrically pumped, C-band Fabry-Perot laser on a silicon photonic integrated circuit by transfer printing", Optics Express, 26(17), 21443 (2018).

[10] Mahmoud, N. Walravens, W. Kuhs, J. Detavernier, C. Hens, Z. Roelkens, G. "Micro-Transfer-Printing of Al2O3Capped Short-Wave-Infrared PbS Quantum Dot Photoconductors", ACS Applied Nano Materials, 8 (2018).

[11] Ye, N. Muliuk, G. Zhang, J. Abbasi, A. Trindade, A. J. Bower, C. Van Thourhout, D. Roelkens, G. "Transfer Print Integration of Waveguide-Coupled Germanium Photodiodes onto Silicon Photonics ICs", IEEE Journal of Lightwave Technology (invited), 36(5), 1249-1254 (2018).

[12] IMEC-EpixFAB (Online). Available: http://www.europractice-ic.com/SiPhotonics_technology_imec_passives.php. Accessed on: Jan. 2019. 